\title{
Corrigenda
}

\section{Overexpression of Protein Kinase $M \zeta$ in the Prelimbic Cortex Enhances the Formation of Long-Term Fear Memory}

Yan-Xue Xue ${ }^{1,6}$, Zhen-Zhen Zhu ${ }^{2,3,6}$, Hai-Bin Han ${ }^{2,3}$, Jian-Feng Liu' ${ }^{1,4,5}$, Shi-Qiu Meng ${ }^{1,4,5}$, Chen Chen ${ }^{1,4,5}$, Jian-Li Yang ${ }^{2,3}$, Ping $W_{u^{\prime}}$ and Lin Lu',4,5

'National Institute on Drug Dependence and Beijing Key Laboratory of Drug Dependence, Peking University, Beijing, China; ${ }^{2}$ Tianjin Medical University, Tianjin, China; ${ }^{3}$ Center of Tianjin Mental Health Center, Tianjin, China; ${ }^{4}$ Institute of Mental Health/Peking University Sixth Hospital and Key Laboratory of Mental Health, Beijing, China; ${ }^{5}$ Peking-Tsinghua Center for Life Sciences and PKU-IDGIMcGovern Institute for Brain Research, Peking University, Beijing, China

Neuropsychopharmacology (2015) 40, 2487. doi:10.1038/npp.2015.136

Correction to: Neuropsychopharmacology advance online publication, 25 March 2015; doi:10.1038/npp.2015.56.
In this article, the positions of the first and second authors (Yan-Xue Xue and Zhen-Zhen Zhu) and the first and second affiliations have been switched.

\section{Sex Moderates the Effects of the Sorll Gene rs2070045 Polymorphism on Cognitive Impairment and Disruption of the Cingulum Integrity in Healthy Elderly}

Ying Liang ${ }^{1,2,6}, \mathrm{He} \mathrm{Li}^{2,3,6}$, Chenlong Lv ${ }^{2,4,6}, \mathrm{Ni}$ Shu ${ }^{1,2}$, Kewei Chen ${ }^{5}, \mathrm{Xin} \mathrm{Li}^{1,2}$, Junying Zhang ${ }^{1,2}$, Liangping $\mathrm{Hu}^{4}$ and Zhanjun Zhang ${ }^{1,2}$

'State Key Laboratory of Cognitive Neuroscience and Learning and IDG/McGovern Institute for Brain Research, Beijing Normal University, Beijing, China; ${ }^{2}$ BABRI Centre, Beijing Normal University, Beijing, China; Institute of Basic Research in Clinical Medicine, China Academy of Chinese Medical Sciences, Beijing, China; ${ }^{4}$ Consulting Center of Biomedical Statistics, Academy of Military Medical Sciences, Beijing, People's Republic of China;

${ }^{5}$ Computational Image Analysis Banner Alzheimer's Institute, Phoenix, AZ, USA2487

Neuropsychopharmacology (20 I5) 40, 2487. doi:I0.1038/npp.20I5.137

Correction to: Neuropsychopharmacology (2015) 40, 1519-1527; doi:10.1038/npp.2015.1; published online 4 February 2015
In this article, the third author, Chenlong Lv, is affiliated only to affiliations number 4 and 6.

\footnotetext{
${ }^{6}$ These authors contributed equally to this work.
} 